\title{
Highly Stable Passive Wireless Sensor for Protease Activity Based on Fatty Acid-Coupled Gelatin Composite Films
}

\section{-Supporting Information-}

Palraj Kalimuthu ${ }^{1,2, \dagger}$, Juan F. Gonzalez-Martinez ${ }^{1,2}$, Tautgirdas Ruzgas ${ }^{1,2}$ and Javier Sotres ${ }^{1,2, *}$

${ }^{1}$ Department of Biomedical Science, Faculty of Health and Society, Malmö University, 20506 Malmö, Sweden

${ }^{2}$ Biofilms-Research Center for Biointerfaces, Malmö University, 20506 Malmö, Sweden

$\dagger$ Present Address: School of Chemistry and Molecular Biosciences, The University of Queensland, St Lucia-4072, Brisbane, Australia

*E-mail: javier.sotres@mau.se

\section{Table of Contents:}

- Section S1: Optical microscopy characterization of SPGEs.

- Section S2: Resonances of reader and transponder antennas.

- Section S3: Optimization of gelatin-based composite composition.

- Section S4: FTIR characterization of gelatin-based composite films.

- Section S5: Stability of the cross-linked gelatin-fatty acid composite in aqueous medium.

- Section S6: Fitting of direct impedance measurements of the proteolytic degradation of gelatin-based composites.

- Section S7: Role of ambient medium in the measured characteristic frequency.

- Section S8: Role of reader-transponder distance/orientation in the measured characteristic frequency.

- Section S9: Wireless monitoring the presence of Proteinase K and Trypsin in aqueous solutions

- References. 

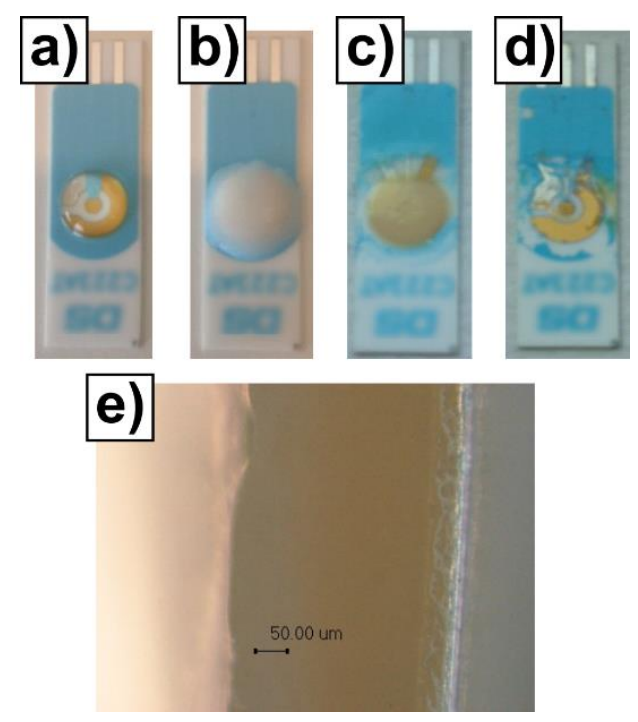

Figure S1. Representative images of screen printed gold electrodes (SPGEs) covered with a) colorless gelatin-glycerol solution, b) gelatin-glycerol-caprylic acid composite, which provided the colorless gelatin-glycerol solution a milky white color, c) gelatin-glycerol-caprylic acid-glutaraldehyde composite, which changed the color of the composite from milky white to yellow. d) Same SPGE as in c) after a proteolytic degradation experiment showing the complete removal of the composite film. e) Representative optical microscopy image of a hydrated gelatin-glycerol-caprylic acid-glutaraldehyde composite film formed on a SPGE showing a thickness of $\sim 350 \mathrm{~nm}$. 


\section{Section S2: Resonances of reader and transponder antennas}

The real and complex part of the impedance of the antenna used for wireless reading in the manuscript is shown below. It can be seen that its resonance is found at $\sim 24 \mathrm{MHz}$, and that the contribution to the resonance found at $\sim 14 \mathrm{MHz}$ (and below) in the wireless measurements presented in the main manuscript can be neglected.
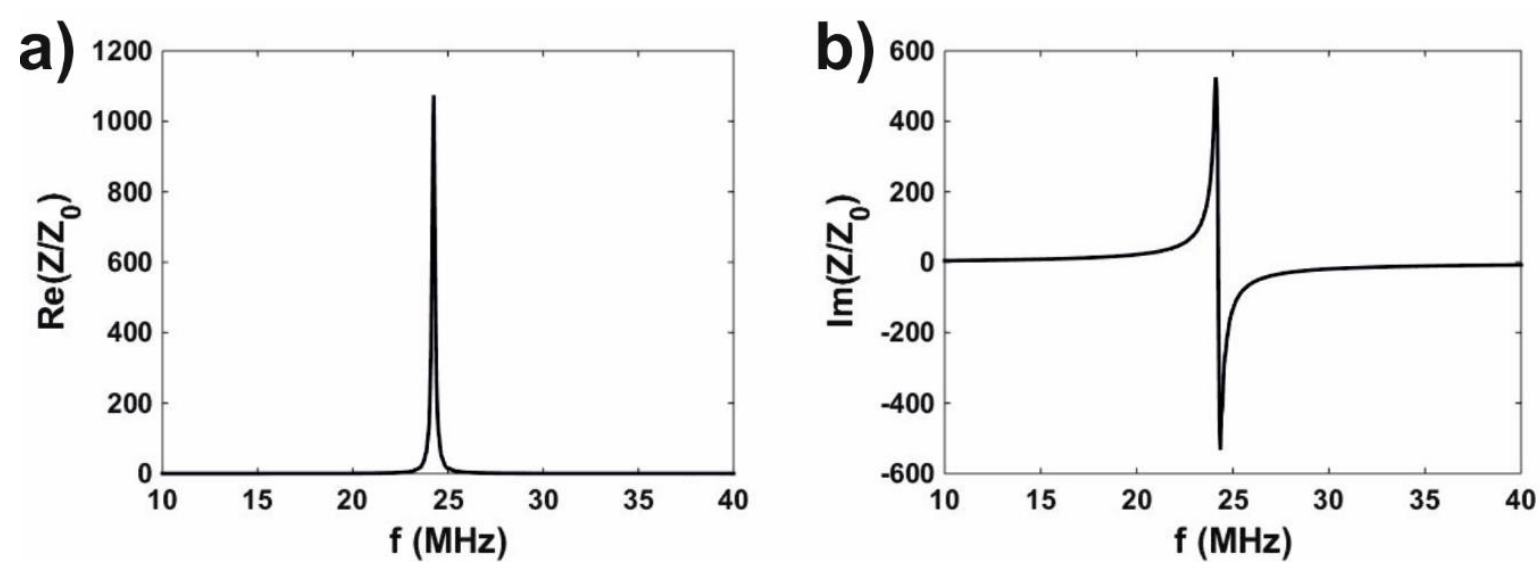

Figure S2. a) Real and b) complex part of the impedance measured for the reader antenna. 


\section{Section S3: Optimization of gelatin-based composite composition}

Cross-linking of gelatin films proved to be essential for performing wireless measurements. Below (Fig. S3A), $\left|S_{11}\right|$ spectra measured for transponders including SPEGs covered with pristine gelatin films and with glutaraldehyde cross-linked gelatin films after being exposed to PBS buffer for $24 \mathrm{~h}$ are shown. For reference, a $\left|S_{11}\right|$ spectrum measured for a transponder including a non-coated SPEG is also shown. It can be clearly observed that the spectra for the transponders including the non-coated SPEG and that including the pristine gelatin coating almost coincide, indicating the poor stability of gelatin films. In contrast, cross-linking the gelatin film with glutaraldehyde provided a well differentiated characteristic frequency.

The effect of incorporating caprylic acid in the cross-linked gelatin films was also systematically studied. As shown below, Fig. S3b, incorporation of caprylic acid incremented the transmitted power (and, therefore, the reading range) at the characteristic frequency. As inferred from the figure, for a $64 \%$ w/v caprylic acid concentration a maximum effect was already achieved.
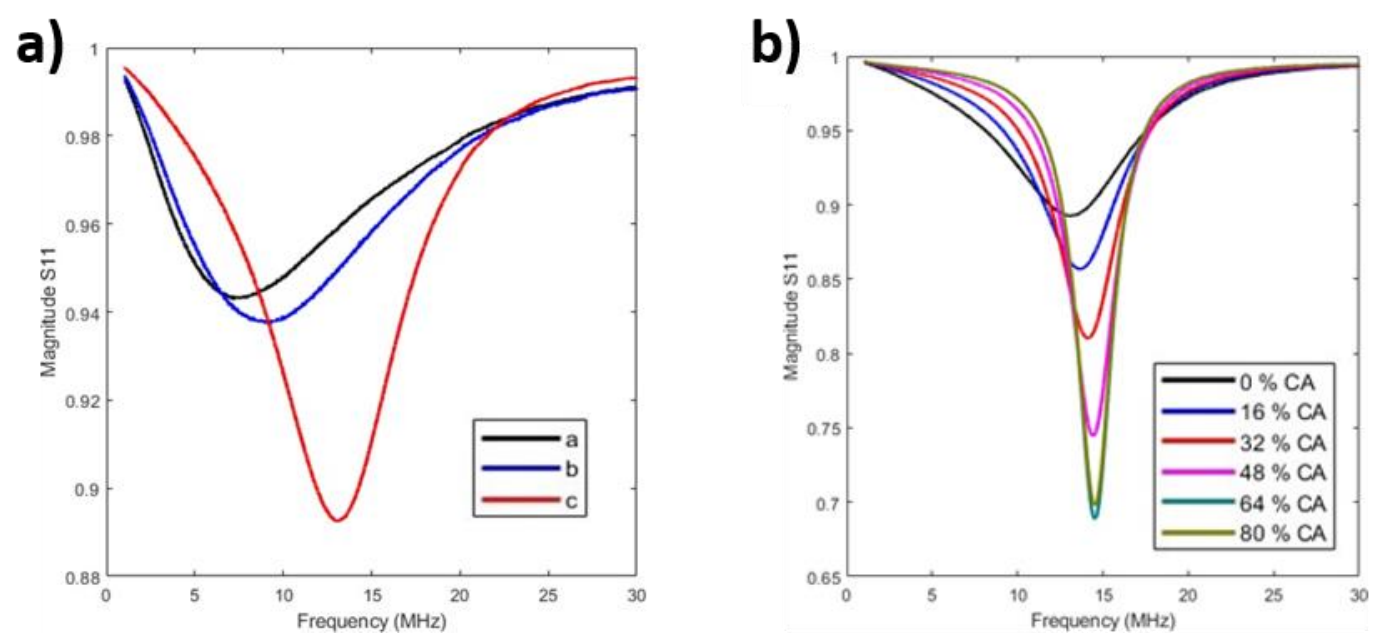

Figure S3. a) $\left|S_{11}\right|$ spectra for (a) bare SPGE, (b) gelatin modified SPGE, (c) GTA cross-linked gelatin modified SPGE. b) $\left|S_{11}\right|$ spectra measured for transponders where the SPGE exposed to PBS buffer was coated with gelatin composites including different concentrations $(\% \mathrm{w} / \mathrm{v})$ of caprylic acid. 


\section{Section S4: FTIR characterization of gelatin-based composite films}

The incorporation of caprylic acid (CA) and glutaraldehyde (GTA) into the pristine gelatin films was examined and confirmed by means of FTIR. FTIR measurements were recorded with a Nicolet 6400 FTIR spectrometer (Thermo Electron Corporation, Waltham MA). The spectrometer was operated at a resolution of $4 \mathrm{~cm}^{-1}$. Each reported spectrum is the sum of 100 individual scans. For analysis, the OMNIC software package was used. All investigated films were coated on glass microscopy slides and dried overnight under vacuum to completely remove absorbed water. Subsequently, the films were peeled and immediately measured in the Attenuated Total Reflection (ATR) FTIR sampling mode. The spectrometer was purged with $\mathrm{N}_{2}$ before and during experiments.

Figure S4a shows FTIR spectra for pristine gelatin (spectrum i), gelatin-CA composite (spectrum ii) and GTA cross linked gelatin-CA composite (spectrum iii) films. Pristine gelatin films displayed, in agreement with previous reports, ${ }^{1-2}$ the characteristic amide A band at $3291 \mathrm{~cm}^{-1}$ for NH stretching coupled with hydrogen bonding, the amide $\mathrm{B}$ band at $2924 \mathrm{~cm}^{-1}$ for $\mathrm{CH}_{2}$ asymmetrical stretching, the amide I peak for $\mathrm{C}=\mathrm{O}$ stretching at $1635 \mathrm{~cm}^{-1}$, the amide II peak for $\mathrm{N}-\mathrm{H}$ bend and $\mathrm{C}-\mathrm{H}$ stretching at $1541 \mathrm{~cm}^{-1}$ and the amide III peak for $\mathrm{C}-\mathrm{N}$ stretch and $\mathrm{N}-\mathrm{H}$ in phase bending at $1234 \mathrm{~cm}^{-1}$. No significant changes in the spectrum were observed upon addition of glycerol (spectrum not shown). This was anticipated as the major bands for glycerol, expected around $849-853 \mathrm{~cm}^{-1}$ (C-C skeletal vibrations), $132-137 \mathrm{~cm}^{-1}(\mathrm{C}-\mathrm{O}$ stretch at $\mathrm{C} 1$ and $\mathrm{C} 3)$ and $1107-1108 \mathrm{~cm}^{-1}(\mathrm{C}-\mathrm{O}$ stretch at $\mathrm{C} 2)$, would coincide with those observed for pristine gelatin. ${ }^{1}$

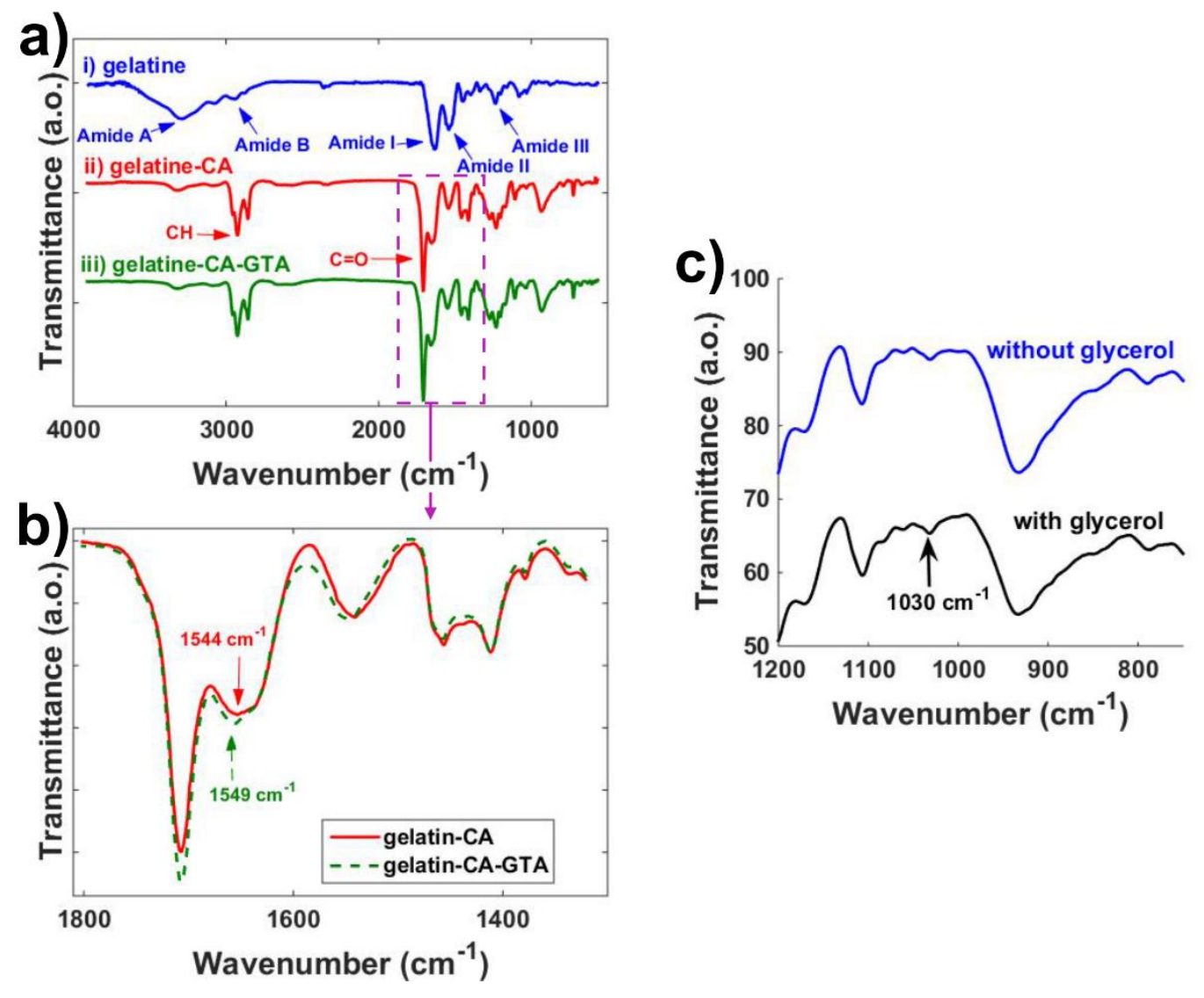

Figure S4. a) FTIR spectra of pristine gelatin, gelatin-CA and GTA cross-linked gelatin-CA composite films. b) gelatin-CA composite film (red solid line) and GTA cross-linked gelatin-CA composite film (green dashed line). c) FTIR spectra of GTA cross-linked gelatin-CA composite films formed with and without glycerol. 
For gelatin-CA composites, bands emerged for $\mathrm{C}=\mathrm{O}$ at $1707 \mathrm{~cm}^{-1}$ and for $\mathrm{C}-\mathrm{H}$ stretching of $\mathrm{CH}_{3}$ at 2925 $\mathrm{cm}^{-1}$ (Fig. S4a, spectrum ii), in agreement with previous report for the incorporation of CA. ${ }^{3}$ Addition of CA immediately provided the colorless gelatin-glycerol solution a milky white color (Supplementary Information Section S1), indicating that the positively charged gelatin residues (arginine, lysine, and histidine) effectively interact with negatively charged CA through electrostatic interaction and formed a homogeneous complex. This is supported by the fact that the amplitude of the amide A band dropped significantly, which can be attributed to the electrostatic interaction between the amine group of gelatin and $\mathrm{COOH}$ group of $\mathrm{CA}$.

Crosslinking the gelatin-CA composite with GTA changed the color of the composited from milky white to yellow (Supplementary Information Section S1). This is associated with the reaction of the free amino groups of gelatin with the aldehyde group $(-\mathrm{CHO})$ of GTA that results in an aldimine linkage $(\mathrm{CH}=\mathrm{N}){ }^{4}$ FTIR also confirmed crosslinking with GTA. Specifically (Fig. S4b), the amide band observed at 1544 $\mathrm{cm}^{-1}$ for un-crosslinked gelatin-CA composites marginally shifted to $1549 \mathrm{~cm}^{-1}$ in agreement with what was previously reported. ${ }^{4}$

We have also measured the FTIR spectrum for a composite film formed in the absence of glycerol and compared it with the spectra for the composite formed with glycerol (Fig. S4c). Usually the $\mathrm{OH}$ band of glycerol appears at a wavelength of $1037 \mathrm{~cm}^{-1}$ whereas the free water band appears at $1030 \mathrm{~cm}^{-1}$. ${ }^{5}$ Both spectra are identical in the range where this band corresponding to glycerol should be observed. The band is actually missing in both spectra and only that associated with free water $\left(1030 \mathrm{~cm}^{-1}\right)$ was observed. Actually, this was expected. We used a $1 \%$ glycerol solution for preparing the composite films and, as reported in, ${ }^{5}$ the $1037 \mathrm{~cm}^{-1}$ glycerol band is only absorbed above $20 \%$ glycerol content. Thus, we cannot confirm from our data the presence of glycerol in the films after exposure to aqueous media. While it might be that the films contain a minor amount of glycerol, most likely glycerol will slowly leach from the films when exposed to aqueous media owing to its solubility. 


\section{Section S5: Stability of the cross-linked gelatin-fatty acid composite in aqueous medium}

The stability of the cross-linked gelatin-fatty acid composite when exposed to PBS buffer was characterized by means of wireless measurements. Figure S5 shows the time evolution of the characteristic frequency obtained from $\left|S_{11}\right|$ spectra for one of these experiments (solid line). It can be seen that the characteristic frequency shift decreased by less than $0.8 \mathrm{MHz}$ for the first $10^{3} \mathrm{~h}$ of exposure.

$\left|S_{11}\right|$ stability of the cross-linked gelatin-fatty acid composite was also monitored during exposure to Lysogeny broth (LB) medium $(10 \mathrm{~g} / \mathrm{L}$ tryptone, $10 \mathrm{~g} / \mathrm{L} \mathrm{NaCl}$ and $5 \mathrm{~g} / \mathrm{L}$ yeast extract in deionized water). As shown in Fig. S5, the characteristic frequency shift decrease during 356h of exposure (dotted line) is far below the $0.8 \mathrm{MHz}$ threshold value used in our study as a protease detection criterion.

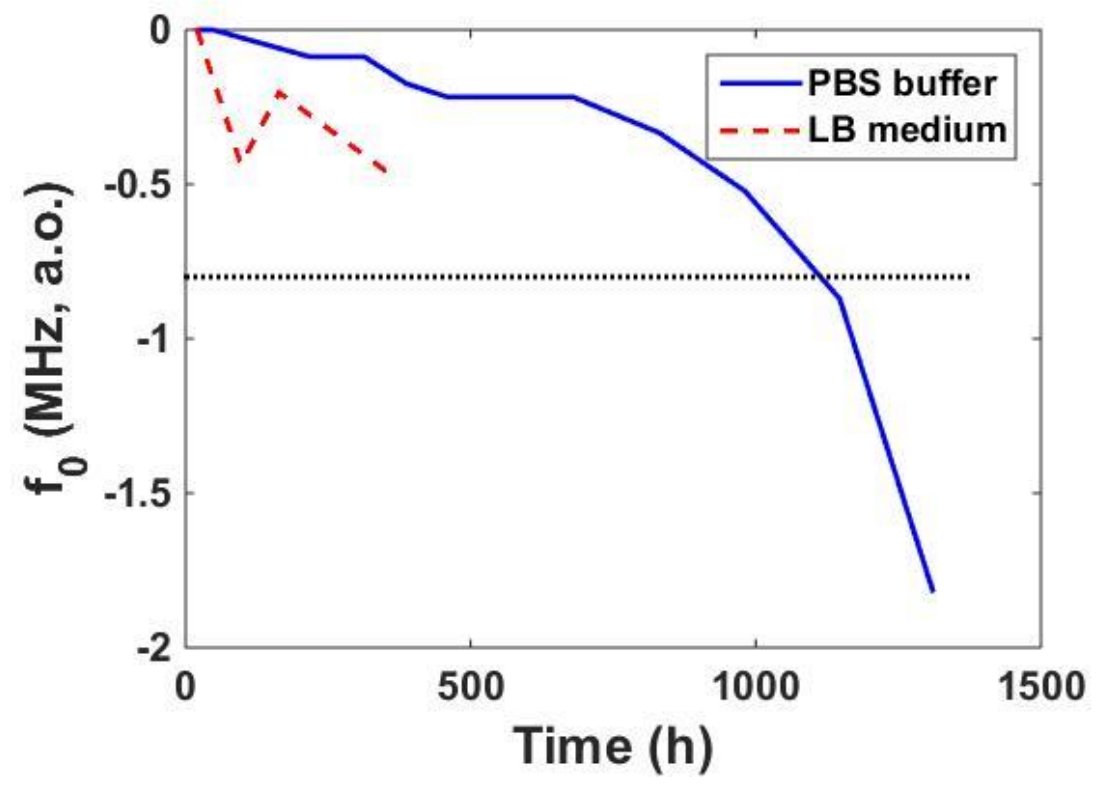

Figure S5. Solid line: Time evolution of the characteristic frequency (arbitrary offset, zero corresponding to the initial measured frequency) obtained from $\left|S_{11}\right|$ spectra measured for a transponder with the gelatin-based composite coated SPEG exposed to PBS buffer (solid line) and LB medium (dashed line). The dotted line highlights the $-0.8 \mathrm{MHz}$ shift used in this work as the threshold for protease detection. 


\section{Section S6: Fitting of direct impedance measurements of the proteolytic degradation of gelatin- based composites}

Direct impedance measurements of the proteolytic degradation of gelatin-based composites were fitted to the impedance of an equivalent circuit consisted of a capacitor and a resistor combined in parallel (Eq. 6 in the manuscript). Representative experimental data for the real and imaginary parts of the impedance, and corresponding fits, for the experiment shown in Fig. 4 of the manuscript are shown in Figure S6. Specifically, Figs. S6a and S6b correspond to the data measured immediately after exposure to the protease solution. Figures S6c and S6d correspond to that measured after 40h i.e., when the composite was totally degraded. It can be seen how the proposed model provided a reasonable good fit for both situations.
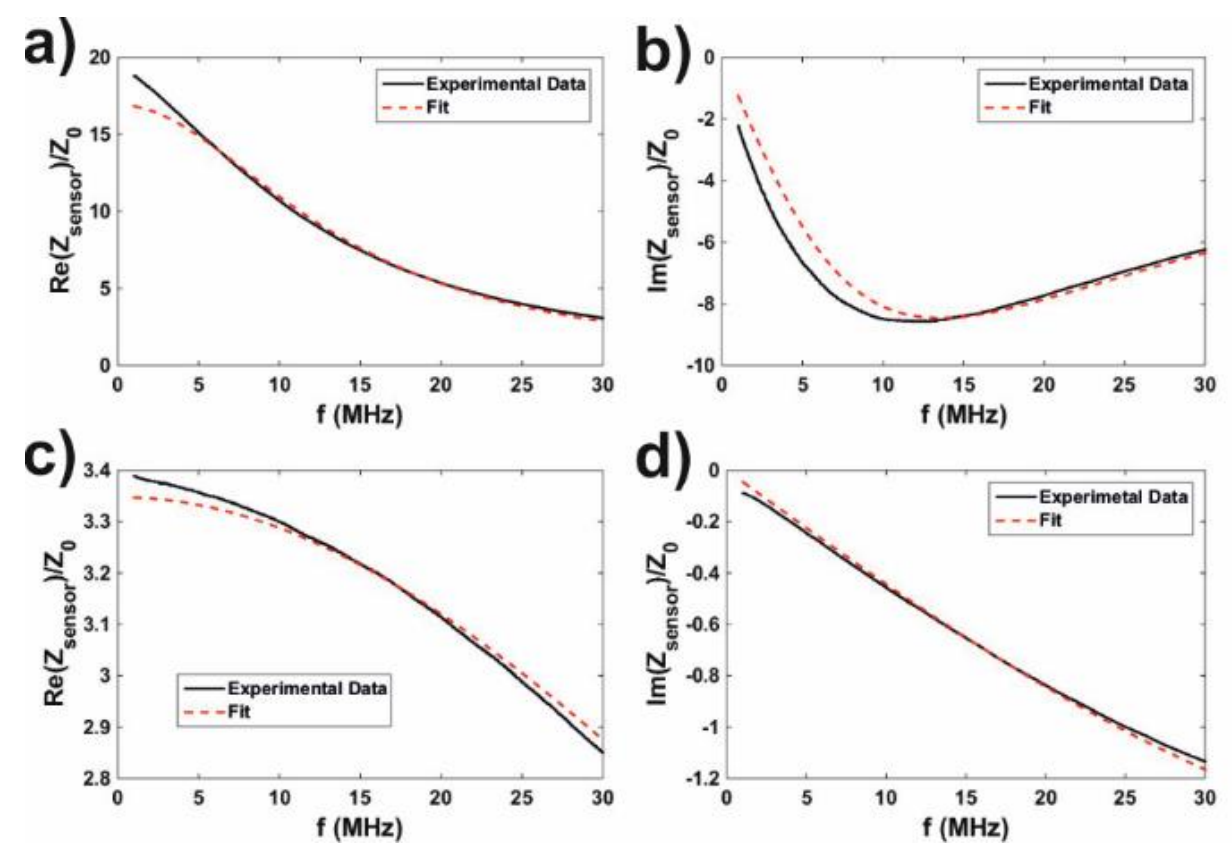

Figure S6. a) Real and b) imaginary components of the impedance measured for a composite-covered SPEG immediately after exposure to a $0.5 \mathrm{mg} / \mathrm{ml}$ solution of proteinase from Aspergillus melleus in PBS buffer. Fits of the experimental data to Eq. 6 of the main manuscript are also shown (dotted lines). c) and d) show similar data, in this case corresponding to $40 \mathrm{~h}$ after being exposed to the protease solution. 
Section S7: Role of ambient medium in the measured characteristic frequency

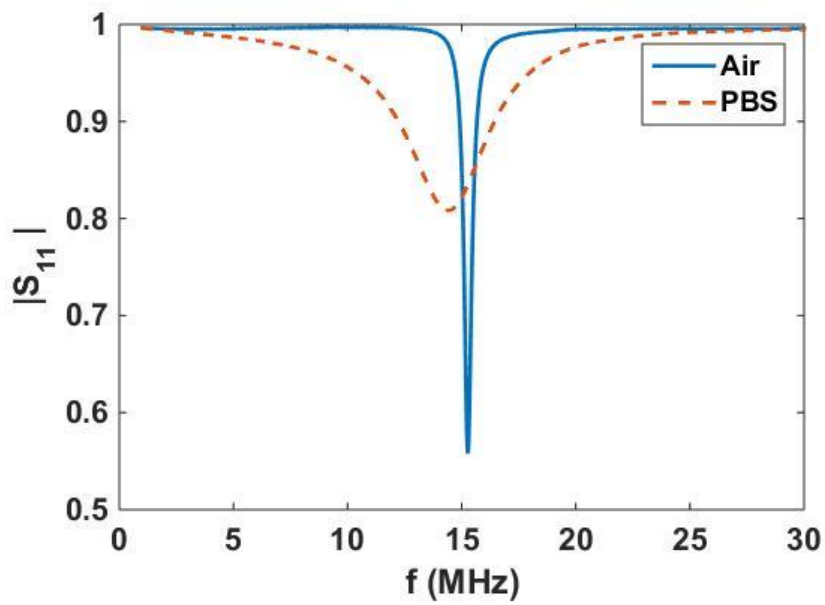

Figure S7. Comparison between the $\left|S_{11}\right|$ spectra measured for the protease sensor in air and PBS buffer. 
The $\left|S_{11}\right|$ spectra for just the antenna of the transponder described in the manuscript (i.e., without the sensor section) was measured at different separations with respect to the reader antenna, but both aligned along in their axial direction (Fig. S8a) and transversally displaced (Fig. S8b). As shown by the figure, the main effect of a change in the relative position between the reader and sensor antennas is a change in the maximum transmitted power. However, the effect on the characteristic frequency i.e., that for which the transmitted power is maximized, is much lower, with changes below $1 \mathrm{MHz}$ (significantly below the enzymatic degradation-induced changes reported in the manuscript).
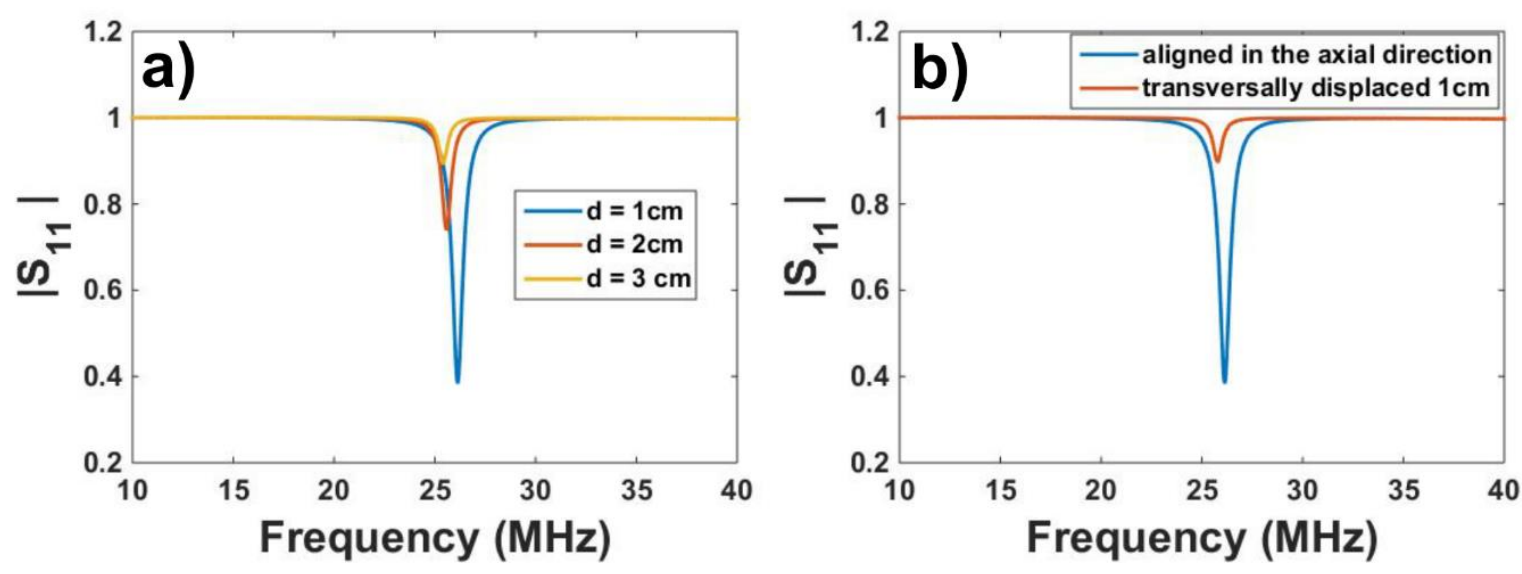

Figure S8. $\left|S_{11}\right|$ spectra for just the antenna of the transponder described in the manuscript (i.e., without the sensor section) measured a) at different separations with respect to the reader antenna, but both aligned along in their axial direction and $\mathbf{b}$ ) transversally displaced a distance of $1 \mathrm{~cm}$ with a separation in the normal direction of also $1 \mathrm{~cm}$. 
Section S9: Wireless monitoring the presence of Proteinase $K$ and Trypsin in aqueous solutions

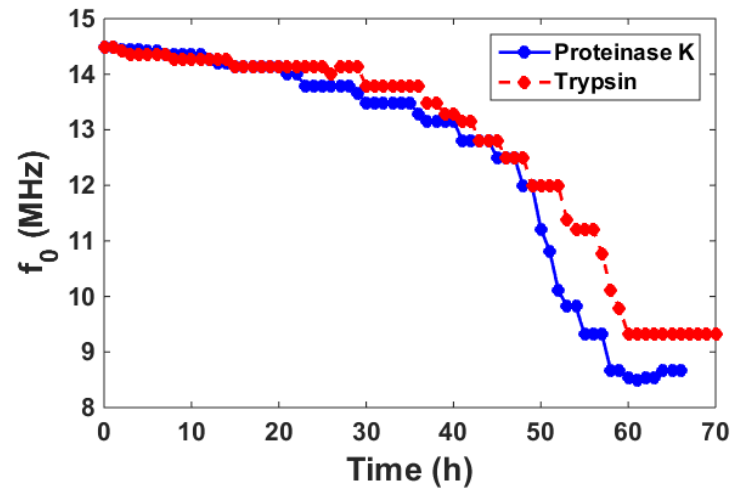

Figure S9. Evolution of the characteristic frequency measured for transponders where the coated SPEG was exposed to $0.05 \mathrm{mg} / \mathrm{ml}$ in PBS solutions of Proteinase K from Tritirachium album and Trypsin. 


\section{References}

(1) Chuaynukul, K.; Nagarajan, M.; Prodpran, T.; Benjakul, S.; Songtipya, P.; Songtipya, L. Comparative Characterization of Bovine and Fish Gelatin Films Fabricated by Compression Molding and Solution Casting Methods. J. Polym. Environ. 2018, 26, 1239-1252.

(2) Nilsuwan, K.; Benjakul, S.; Prodpran, T. Influence of palm oil and glycerol on properties of fish skin gelatin-based films. J. Food Sci. Technol. 2016, 53, 2715-2724.

(3) Konuklu, Y.; Paksoy, H. O. The Preparation and Characterization of Chitosan-Gelatin Microcapsules and Microcomposites with Fatty Acids as Thermal Energy Storage Materials. Energy Technol. 2015, 3, 503-508.

(4) Farris, S.; Song, J.; Huang, Q. Alternative Reaction Mechanism for the Cross-Linking of Gelatin with Glutaraldehyde. J. Agric. Food Chem. 2010, 58, 998-1003.

(5) Nor, M. H. M.; Nazmi, N. N. M.; Sarbon, N. M. Effects of plasticizer concentrations on functional properties of chicken skin gelatin films. Int. Food Res. J. 2017, 25, 1910-1918 